CERN-TH-7204/94

March, 1994

hep-th/9403155

\title{
On Non-Abelian Duality
}

\author{
Enrique Álvarez ${ }^{1}$, Luis Álvarez-Gaumé and Yolanda Lozano ${ }^{1}$ \\ Theory Division CERN \\ 1211 Geneva 23 \\ Switzerland
}

\begin{abstract}
A general study of non-abelian duality is presented. We first identify a possible obstruction to the conformal invariance of the dual theory for non-semisimple groups. We construct the exact non-abelian dual for any Wess-Zumino-Witten (WZW) model for any anomaly free subgroup, and the corresponding extension for coset conformal field theories. We characterize the exact non-abelian dual for $\sigma$-models with chiral isometries and extend the standard notion of duality to anomalous subgroups of WZW-models, thus giving a way of constructing dual transformations for different groups on the left and on the right. We also present some new examples of non-abelian duality for four-dimensional gravitational instantons.
\end{abstract}

\footnotetext{
${ }^{1}$ On leave of absence from: Departamento de Física Teórica, Universidad Autónoma de Madrid, 28049 Madrid, Spain
} 


\section{Introduction}

Duality transformations with respect to abelian (discrete or continuous) symmetries have a long history in statistical mechanics [1]. In String Theory and two-dimensional Conformal Field Theory abelian duality with respect to continuous symmetries has received an increasing amount of attention [2]. Of more recent history is the notion of non-abelian duality [3, 4], which has no analogue in statistical mechanics. The basic idea of [3], partly inspired in the treatment of abelian duality presented in [6], is to consider a conformal field theory with a non-abelian symmetry group $G$. Particularly one considers a $\sigma$-model on a manifold $M$ with isometry group $G$. The duality transformation proceeds in two steps: i) First one gauges the isometry group, thus introducing some gauge field variables $A_{\mu}^{a}$. The gauge field is required to be flat; and this is implemented by adding to the lagrangian a term of the form $\chi_{a} F_{\mu \nu}^{a} \epsilon^{\mu \nu}$, where $\chi_{a}$ is a lagrange multiplier imposing the flatness constraint on the gauge connection. It is naively clear that if we first perform the integral ovel $\chi_{a}$, this provides a $\delta$-function $\delta\left(F^{a}\right)$ on the measure, which in turn implies that $A_{\mu}=g^{-1} \partial_{\mu} g$ is a pure gauge (we consider a spherical world-sheet for simplicity). Using the original symmetry of the theory we can absorb the gauge field by a change of variables on the manifold. In this way we recover the original model. ii) The second step consists of integrating first the gauge field $A_{\mu}^{a}$. Since we have no gauge kinetic term, the integration is gaussian, and we obtain a lagrangian, $\tilde{L}(\phi, \chi)$, depending on the original variables $\phi^{i}$ and the auxiliary variables $\chi_{a}$, which is still gauge invariant. Fixing finally the gauge we obtain the dual theory. In the abelian case it is also possible to work out the mapping between operators for the original and the dual theory, as well as the global topology of the dual manifold $[2,4]$. In [6] it was further shown that if one starts with a conformal field theory (CFT), conformal invariance is preserved by abelian duality. The proof was based on an analogy between the duality transformation and the GKO construction [7]. Thus for $G$ abelian we have a rather thorough understanding of the detailed local and global properties of duality. Up till now our knowledge of the non-abelian case was far more limited, and it is one of the purposes of this paper to mend this situation.

An interesting example of non-abelian duality recently studied is the one presented in [8]. They considered a cosmological solution to string theory of Bianchi type $\mathrm{V}$. In fact to satisfy the $\beta$-function constraints for conformal 
invariance, the space is flat. It is the interior of the light-cone through the origin in Minkowski space. They then performed the non-abelian duality transformation with respect to a non-semisimple subgroup of the Lorentz group, and to their surprise they found that the dual model did not satisfy conformal invariance. We will show that when one analyzes carefully the measure of integration over the gauge fields and its dependence on the world sheet metric, one encounters a mixed gauge and gravitational anomaly [9] when any generator of $G$ in the adjoint representation has a non-vanishing trace $^{2}$. This only happens for non-semisimple groups. This mixed anomaly generates a contribution to the trace anomaly which cannot be absorbed in a dilaton shift. This explains why the dual model in [8] violates conformal invariance, and imposes a mild anomaly cancellation condition for the consistency of non-abelian duality.

The organization of this paper is as follows: In section two we briefly summarize the operations involved in explicitly computing the abelian or non-abelian dual of a given theory. We will show that one can understand the transformations as a change of gauge condition, and this will make clear that one of the possible obstructions to conformal invariance is found in mixed two-dimensional anomalies. In section three we compute the dependence of the gauge field measure on the world-sheet metric. We evaluate the leading terms in the effective action, and we explicitly show the existence of mixed anomalies when some generators of the adjoint representation of the isometry group are not trace-free. Following the approach developed in this section, we present in section four an exact treatment of the non-abelian duality transformation for Wess-Zumino-Witten (WZW) models [10] with group $G$ with respect to any vector subgroup of $G \times G$. If $k$ is the level of the WZW model, the non-abelian dual with respect to $H \subset G_{D}$ (where $G_{D}$ is the diagonal embedding of $G)$ is given by $(G / H)_{k} \times H_{k}$ where $(G / H)_{k}$ is the coset GKO construction for $G$ and $H$ [7] and $H_{k}$ is a WZW theory for $H$ with level $k$ (assuming for simplicity that the Dynkin index for the embedding $H \subset G$ is 1 ). In this way we also find the global topology of the non-abelian dual. The extension of our construction to the coset conformal field theory $G / H$ when $H$ has a non-trivial centralizer in $G$ is straightforward. In section

\footnotetext{
${ }^{2}$ The breakdown of conformal invariance for non-semisimple groups due to the possible existence of generators in the adjoint representation which are not trace free was anticipated in [5] in the note added.
} 
five we consider a general $\sigma$-model with left and right chiral non-abelian currents, and show that the non-abelian dual with respect to any vector subgroup is again described by a generalized GKO construction coupled to a WZW theory determined by the auxiliary field $\chi$. This will give us the clue of how to generalize duality in a heterotic fashion even with respect to anomalous isometry groups. The auxiliary field is given transformation properties to cancel the anomaly as in the abelian case. We then make some remarks on the general case where the isometry currents are not necessarily chiral. Once again the key to the exact treatment of non-abelian duality lies on the coupling to a WZW theory. One of the basic ingredients is a way of writing the term $\chi_{a} F_{\mu \nu}^{a} \epsilon^{\mu \nu}$ (which always appears in duality transformations) in terms of WZW lagrangians. In section six we present some interesting nonabelian duality transformations for the Eguchi-Hanson and Euclidean TaubNUT gravitational instantons. We finish with the conclusions and outlook in section seven.

\section{Duality transformations and gauge equiva- lence}

We work throughout with light-cone variables in two dimensions, $x^{ \pm}=\tau \pm \sigma$. Consider first a $\sigma$-model with an isometry generated by the Killing vector $\xi^{i}$

$$
L_{0}=\frac{1}{2 \pi} g_{i j}(\phi) \partial_{+} \phi^{i} \partial_{-} \phi^{j}
$$

and

$$
£(\xi) g_{i j}=\nabla_{i} \xi_{j}+\nabla_{j} \xi_{i}=0
$$

Gauging the isometry and adding a Lagrange multiplier $\chi$ to force the gauge connection to be flat we obtain the starting point of any duality transformation:

$$
\begin{gathered}
L=\frac{1}{2 \pi} g_{i j}(\phi) D_{+} \phi^{i} D_{-} \phi^{j}+\frac{1}{2 \pi} \chi\left(\partial_{+} A_{-}-\partial_{-} A_{+}\right) \\
D_{ \pm} \phi^{i}=\partial_{ \pm} \phi^{i}+\xi^{i}(\phi) A_{ \pm}
\end{gathered}
$$

If we integrate first over $\chi$, we obtain (on the sphere) $A_{ \pm}=\partial_{ \pm} \alpha$. Then we make a change of variables on $\phi^{i}$ to eliminate $\alpha$. This is done as follows: The 
$\sigma$-model is defined on a manifold $(M, g)$, the metric being constant along the orbits of the isometry group, $G$. The action of $G$ on $M$ is represented by a function $\phi^{\prime} i=f^{i}(\phi, \alpha)$. Changing variables from $\phi^{i}$ to $\phi^{\prime i}$ brings the action into the original form with respect to the $\phi^{\prime}$ variables. Since the path integral measure contains a factor of $(\operatorname{det} g)^{\frac{1}{2}}$ at each point, i.e., $\prod_{\sigma, \tau} \mathcal{D} \phi(\tau, \sigma)(\operatorname{detg}(\phi(\tau, \sigma)))^{\frac{1}{2}}$ one indeed recovers the same quantum theory we started with. If we now integrate over the gauge fields, we obtain the dual action. Choosing coordinates adapted to $\xi^{i}, \phi^{i}=\left(\theta, \phi^{\alpha}\right), \xi^{i} \frac{\partial}{\partial \phi^{i}}=\frac{\partial}{\partial \theta}$, the dual lagrangian is [2]:

$$
\tilde{L}=\frac{1}{2 \pi}\left(\tilde{g}_{i j}(\tilde{\phi})+\tilde{b}_{i j}(\tilde{\phi})\right) \partial_{+} \tilde{\phi}^{i} \partial_{-} \tilde{\phi}^{j}
$$

with $\tilde{\phi}^{0}=\chi, \tilde{\phi}^{\alpha}=\phi^{\alpha}$, and

$$
\begin{aligned}
\tilde{g}_{00} & =\frac{1}{g_{00}} \\
\tilde{g}_{\alpha \beta} & =g_{\alpha \beta}-\frac{g_{0 \alpha} g_{0 \beta}}{g_{00}} \\
\tilde{b}_{0 \alpha} & =\frac{1}{g_{00}} g_{0 \alpha} \\
\tilde{b}_{\alpha \beta} & =0 .
\end{aligned}
$$

We can look at this transformation differently. Since the lagrangian is invariant under

$$
\begin{aligned}
\delta \phi^{i} & =\epsilon \xi^{i}(\phi) \\
\delta A_{ \pm} & =-\partial_{ \pm} \epsilon
\end{aligned}
$$

we can choose the light-cone gauge, $A_{+}=0$, yielding

$$
L=L_{0}+\frac{1}{2 \pi} \xi_{i}(\phi) \partial_{+} \phi^{i} A_{-}+\chi \partial_{+} A_{-}
$$

ignoring the ghost contribution. The integration over $A_{-}$gives a $\delta$-function imposing $\partial_{+} \chi-\xi_{i} \partial_{+} \phi^{i}=0$. The integration over $\chi$ is now trivial. Hence our lagrangian in the $A_{+}=0$ gauge is equivalent to the original model. If we now choose instead the Landau gauge $\partial_{\mu} A^{\mu}=0$, we can parametrize (on 
the sphere) $A_{\mu}=\epsilon_{\mu \nu} \partial^{\nu} \rho$; that is, $A_{ \pm}= \pm \partial_{ \pm} \rho$. The lagrangian now reads in adapted coordinates:

$$
\begin{aligned}
L & =g_{00}\left(\partial_{+} \theta+\partial_{+} \rho\right)\left(\partial_{-} \theta-\partial_{-} \rho\right) \\
& +g_{0 \alpha}\left(\partial_{+} \theta+\partial_{+} \rho\right) \partial_{-} \phi^{\alpha} \\
& +g_{0 \alpha}\left(\partial_{-} \theta-\partial_{-} \rho\right) \partial_{+} \phi^{\alpha} \\
& +g_{\alpha \beta} \partial_{+} \phi^{\alpha} \partial_{-} \phi^{\beta}+\partial_{+} \chi \partial_{-} \rho+\partial_{-} \chi \partial_{+} \rho
\end{aligned}
$$

After some simple manipulations we obtain:

$$
\begin{aligned}
2 \pi L & =g_{00}\left(\partial_{+} \theta+\partial_{+} \rho+\frac{1}{g_{00}}\left(j_{+}-\partial_{+} \chi\right)\right)\left(\partial_{-} \theta-\partial_{-} \rho+\frac{1}{g_{00}}\left(j_{-}+\partial_{-} \chi\right)\right) \\
& -\left(\partial_{+} \theta \partial_{-} \chi-\partial_{-} \theta \partial_{+} \chi\right)+\frac{1}{g_{00}} \partial_{+} \chi \partial_{-} \chi \\
& +\left(g_{\alpha \beta}-\frac{g_{0 \alpha} g_{0 \beta}}{g_{00}}\right) \partial_{+} \phi^{\alpha} \partial_{-} \phi^{\beta} \\
& -\frac{g_{0 \alpha}}{g_{00}}\left(\partial_{+} \phi^{\alpha} \partial_{-} \chi-\partial_{-} \phi^{\alpha} \partial_{+} \chi\right)
\end{aligned}
$$

where $j_{ \pm}=g_{0 \alpha} \partial_{ \pm} \phi^{\alpha}$. Once again we have ignored the Faddeev-Popov ghosts. The second term in the preceding equation is purely topological and it sets the global range of the auxiliary field $\chi$. The last three terms give the dual lagrangian, whereas the first term can be eliminated by integrating over $(\theta, \rho)$, yielding a determinant of $1 / g_{00}$, which determines the duality properties of the dilaton, if one defines it in a convenient way, as done by Buscher [2].

If the theory we started with is gauge invariant at the quantum level, then a change of gauge is not a change in physics, and both models are equivalent. This argument has the advantage of holding even for theories which are not conformally invariant. It is also clear that the same construction applies to non-abelian duality. Within this point of view, possible problems with duality in CFT should be found in the interplay between the trace or the gravitational anomaly with the gauge anomaly, and this will be our main theme in section three.

For future reference we collect here all the basic formulae for non-abelian duality. This reduces to the construction of a gauge $\sigma$-model with arbitrary WZW-term, where we gauge the isometry group $G$. This has been carried out in $[12,13]$. The general form of a WZW $\sigma$-model on a manifold $M$ with 
metric $g_{i j}$ is:

$$
S[\phi]=\frac{1}{4 \pi} \int_{M_{2}} d x^{+} d x^{-} g_{i j}(\phi) \partial_{+} \phi^{i} \partial_{-} \phi^{j}+\frac{1}{12 \pi} \int_{B_{3}, \partial B_{3}=M_{2}} H
$$

$\mathrm{H}$ is a three-form on $M$ which belongs to the integral cohomology of $M$, and we assume it normalized so that

$$
\frac{1}{24 \pi^{2}} \int_{C_{3}} H=\text { integer }
$$

where $C_{3}$ is a 3 -cycle in $M . B_{3}$ is a three-dimensional space whose boundary is $M_{2}$. If $M_{2}$ has a trivial topology, $B_{3}$ is topologically a three-ball. However, if $M_{2}$ is a Riemann surface of genus $g$ (so that $x^{ \pm}$are complex coordinates), then $B_{3}$ is a genus g handlebody. The measure $d x^{-} \wedge d x^{+}=2 d \tau \wedge d \sigma$ is chosen for convenience. The normalization $1 / 4 \pi$ implies that the two-point function for the massless scalar field is $-\log \left(x^{+}-y^{+}\right)\left(x^{-}-y^{-}\right)$. The isometry group $G$ of $M$ is generated by the Killing vectors $\xi_{a}{ }^{i}(a=1 \ldots \operatorname{dim} \mathrm{G})$. Introducing gauge fields $A_{ \pm}{ }^{a}$, the gauge transformations are:

$$
\begin{aligned}
& \delta \phi^{i}=\epsilon^{a}(x) \xi_{a}{ }^{i}(\phi) \\
& \delta A_{ \pm}^{a}=\partial_{ \pm} \epsilon^{a}+f^{a}{ }_{b c} A_{ \pm}{ }^{b} \epsilon^{c} \\
& D_{ \pm} \phi^{i}=\partial_{ \pm} \phi^{i}-\xi_{a}{ }^{i} A_{ \pm}{ }^{a}
\end{aligned}
$$

and $f^{a}{ }_{b c}$ are the structure constants of the group

$$
\left[\xi_{a}, \xi_{b}\right]^{i}=\left(\xi_{a}{ }^{k} \frac{\partial \xi_{b}^{i}}{\partial \phi^{k}}-\xi_{b}{ }^{k} \frac{\partial \xi_{a}^{i}}{\partial \phi^{k}}\right)=f_{a b}{ }^{c} \xi_{c}{ }^{i}
$$

Global invariance of the action implies some conditions on $g_{i j}$ and $H_{i j k}$. On the metric the conditions are just the Killing equations. The invariance of the torsion is, in turn, conveyed by the equations

$$
\mathcal{L}\left(\xi_{a}\right) H=0
$$

Since $H$ is closed, and acting on forms $\mathcal{L}\left(\xi_{a}\right)=i\left(\xi_{a}\right) d+d i\left(\xi_{a}\right)\left(i\left(\xi_{a}\right)\right.$ being the interior product, or contraction with the vector field $\xi_{a}$ ), the invariance now requires that for every $\xi_{a}$ we should be able to find a one-form globally defined on $M$ and satisfying $i\left(\xi_{a}\right) H=d v_{a}$. In components:

$$
\xi_{a}^{i} H_{i j k}=\partial_{j} v_{a k}-\partial_{k} v_{a j}
$$


We need furthermore the condition that the $v_{a i}$ form a representation of the isometry group

$$
\mathcal{L}\left(\xi_{a}\right) v_{b i}=\xi_{a}{ }^{k} \partial_{k} v_{b i}+v_{b k} \partial_{i} \xi_{a}{ }^{k}=f_{a b}^{c} v_{c i}
$$

The simplest way to gauge the preceding action is to use Noether's procedure. We first perform a variation of the ungauged action. Since the parameters are point-dependent, there will be terms in it proportional to $\partial_{+} \epsilon^{a}$ and $\partial_{-} \epsilon^{a}$ :

$$
4 \pi \delta S[\phi]=\int \partial_{+} \epsilon^{a}\left(\xi_{a i}+v_{a i}\right) \partial_{-} \phi^{i}+\partial_{-} \epsilon^{a}\left(\xi_{a i}-v_{a i}\right) \partial_{+} \phi^{i}
$$

Note in passing that the condition to have a left-chiral current $\left(\partial_{-} J_{+a}=0\right)$ is $\xi_{a}+v_{a}=0$; similarly a right chiral current requires $\xi_{a}-v_{a}=0$. To cancel our variation, we add a term to the action linear in the gauge fields $A_{ \pm}{ }^{a}$. In turn this new contribution to the action will have a gauge variation, which we try to cancel by adding another term to the action which is quadratic in $A_{ \pm}$. At this point one finds that the variation of the full action is independent of the $\phi$-fields and it is equal to the two dimensional gauge anomaly. The gauged action $[12,13]$ becomes:

$$
\begin{aligned}
4 \pi S[\phi, A] & =\int_{M_{2}} d x^{-} d x^{+} g_{i j}(\phi) D_{+} \phi^{i} D_{-} \phi^{j}+\frac{1}{3} \int_{B_{3}} H_{i j k} d \phi^{i} d \phi^{j} d \phi^{k} \\
& -\int\left(A_{+}^{a} v_{a i} \partial_{-} \phi^{i}-A_{-}^{a} v_{a i} \partial_{+} \phi^{i}\right)+\int c_{[a b]} A_{+}^{a} A_{-}^{b}
\end{aligned}
$$

where

$$
c_{a b}=v_{a i} \xi_{b}^{i}, c_{(a b)}=\frac{1}{2}\left(c_{a b}+c_{b a}\right), c_{[a b]}=\frac{1}{2}\left(c_{a b}-c_{b a}\right),
$$

and under a gauge variation:

$$
\delta S[\phi, A]=-\frac{1}{4 \pi} \int c_{(a b)}\left(A_{+}^{a} \partial_{-} \epsilon^{b}-A_{-}^{a} \partial_{+} \epsilon^{b}\right),
$$

The coefficients $c_{(a b)}$ are constant:

$$
d c_{a b}=d\left(v_{a} \cdot \xi_{b}\right)=d\left(i\left(\xi_{b}\right) \cdot v_{a}\right)=\mathcal{L}\left(\xi_{b}\right) \cdot v_{a}-i\left(\xi_{b}\right) i\left(\xi_{a}\right) H
$$

after symmetrizing $(a, b)$ the result vanishes. Therefore to assure gauge invariance we must require $(2.20)$ to vanish, i.e. $c_{(a b)}=0$. If this condition 
is satisfied, the dual action is obtained by adding to $S[\phi, A]$ the Lagrange multiplier enforcing A to be flat:

$$
\tilde{S}[\phi, A]=S[\phi, A]+\frac{1}{2 \pi} \int \chi_{a} F_{+-}^{a}(A) .
$$

From $(2.18,21)$ we see that the gauge field appears at most quadratically in the action. We can thus eliminate A by using its equations of motion up to a jacobian factor which affects the dilaton transformation rules [2, 3].

One of the difficulties in understanding the global properties of the dual action lies in the fact that apparently there is no topology in the auxiliary field $\chi$. We will show in the next few sections that $\chi$ is actually described by a WZW-model based on the group $G$ used to dualize. To conclude we remark that if in (2.21) we integrate first over $\chi^{a}$, we obtain that $A_{ \pm}=g^{-1} \partial_{ \pm} g$. Making a change of variables, as described after equation (2.4) $\phi^{i} \rightarrow \phi^{\prime}{ }^{i}=$ $f^{i}(\phi, g)$, where $f$ describes the action of the group on the manifold $\mathrm{M}$, we get back to the original theory.

There is a remarkable identity proved by Hull and Spence [12] which will be useful later on. The last three terms in (2.11) can be expressed in terms of a 3-form. Define the covariant differentials:

$$
\begin{aligned}
D \phi^{i} & \equiv d \phi^{i}-\xi_{a}^{i} A^{a} ; \\
A^{a} & =A_{\mu}^{a} d x^{\mu} \\
F^{a} & =d A^{a}-\frac{1}{2} f_{b c}^{a} A^{b} A^{c} ;
\end{aligned}
$$

Then after some algebra using $(2.15,16)$ the action becomes:

$$
\begin{aligned}
4 \pi S[\phi, A] & =\int_{M_{2}} g_{i j}(\phi) D_{+} \phi^{i} D_{-} \phi^{j}+\int_{B_{3}}\left(\frac{1}{3} H_{i j k} D \phi^{i} D \phi^{j} D \phi^{k}+v_{a i} D \phi^{i} F^{a}\right. \\
& \left.+c_{(a b)} A^{a} d A^{b}+\frac{1}{3} f_{m n}^{b} c_{(a b)} A^{m} A^{n} A^{a}\right)
\end{aligned}
$$

All terms but the last two are gauge invariant, and the last two are a ChernSimons term whose gauge variation is responsible for the anomaly (2.20).

The lesson to be learned from this section is that one way to think about duality is the equivalence of descriptions of the same theory based on different gauges. Hence in trying to carefully construct the quantum properties of the dual theory we have to understand the interplay between gauge invariance and conformal invariance. This we do in the next section. 


\section{Mixed anomalies and effective actions}

To solve the puzzle posed by the example studied in [8] we need to analyze carefully the integration measure over the gauge fields for the action (2.18). The authors of [8] considered a four-dimensional Bianchi V cosmological solution to string theory. The $b_{\mu \nu}$ field vanishes, and the metric takes the form:

$$
d s^{2}=-d t^{2}+a(t)^{2}\left(d x^{2}+e^{-2 x}\left(d y^{2}+d z^{2}\right)\right)
$$

Conformal invariance requires $a(t)=t$, in which case the metric is flat: It is the interior of the light-cone in Minkowski space. This can be seen by first making the change of variables $x \rightarrow \ln x$,

$$
d s^{2}=-d t^{2}+\frac{t^{2}}{x^{2}}\left(d x^{2}+d y^{2}+d z^{2}\right)
$$

with $x>0, y, z \in R$. The equal t-surfaces are described by the upper halfspace with constant negative curvature. By a simple change of variables we can transform the upper half-space into the mass hyperboloids in Minkowski space. Hence this theory is indeed conformally invariant. The group of motions on the equal time surfaces is given by:

$$
\begin{aligned}
& P_{0}=\frac{\partial}{\partial x}+y \frac{\partial}{\partial y}+z \frac{\partial}{\partial z} \\
& P_{1}=\frac{\partial}{\partial y}, \quad P_{2}=\frac{\partial}{\partial z}
\end{aligned}
$$

with commutation relations:

$$
\left[P_{0}, P_{1}\right]=-P_{1}, \quad\left[P_{0}, P_{2}\right]=-P_{2}, \quad\left[P_{1}, P_{2}\right]=0
$$

The only non-vanishing structure constants are:

$$
f_{01}^{1}=f_{02}^{2}=-1
$$

Since the adjoint representation is given by $\left(T_{a}\right)_{b}^{c}=-f_{a b}^{c}$, we easily see that $\operatorname{Tr} T_{0}=2, \operatorname{Tr} T_{1}=\operatorname{Tr} T_{2}=0$, therefore the group is not semisimple. It is worth noting that the group generated by (3.3) must be a subgroup of the Lorentz group $\mathrm{SO}(3,1)$. Indeed, if $K_{i} \mathrm{i}=1,2,3$ are the boost generators and 
$J_{i} \mathrm{i}=1,2,3$ are the rotation generators, it is easy to see that (3.3) is satisfied with the identifications:

$$
P_{0}=K_{3}, \quad P_{1}=J_{1}-K_{2}, \quad P_{2}=J_{2}+K_{1} .
$$

If one performs the duality transformation for (3.3) according to the rules in [3], then one finds [8] that the dual theory does not satisfy the $\beta$-function equations to first order in $\alpha^{\prime}$ no matter what choice one makes for the dilaton field $\Phi[14]$ :

$$
\begin{gathered}
R_{\alpha \beta}-\frac{1}{4} H_{\alpha \beta}^{2}-\nabla_{\alpha} \nabla_{\beta} \Phi=0 \\
-\frac{1}{2} \nabla^{\gamma} H_{\alpha \beta \gamma}-\frac{1}{2} \nabla^{\gamma} \Phi H_{\alpha \beta \gamma}=0 \\
R-\frac{1}{12} H^{2}-2 \nabla^{2} \Phi-(\nabla \Phi)^{2}-2 \frac{c-d}{3 \alpha^{\prime}}=0
\end{gathered}
$$

where

$$
H_{\alpha \beta \gamma}=\nabla_{[\alpha} b_{\beta \gamma]}, H_{\alpha \beta}^{2}=H_{\alpha \rho \sigma} H_{\beta}^{\rho \sigma} .
$$

A similar example along the same lines is provided by a Bianchi IV type cosmological model with metric:

$$
d s^{2}=-d t^{2}+a^{2}(t) d x^{2}+b^{2}(t) e^{-x}\left(d y^{2}+d z^{2}\right) .
$$

Conformal invariance to order $\alpha^{\prime}$ requires $a(t)=t / 2, b(t)=t$; and the group of motions is generated by

$$
K_{1}=\frac{\partial}{\partial y}, \quad K_{2}=\frac{\partial}{\partial z}, \quad K_{3}=\frac{\partial}{\partial x}+y \frac{\partial}{\partial y},
$$

with commutation relations

$$
\left[K_{1}, K_{2}\right]=0, \quad\left[K_{2}, K_{3}\right]=0, \quad\left[K_{1}, K_{3}\right]=K_{1} .
$$

Now $\operatorname{Tr} K_{3}^{\text {adj }}=1, \operatorname{Tr} K_{1}^{\text {adj }}=\operatorname{Tr} K_{2}^{\text {adj }}=0$, and as in the previous example the non-abelian dual of (3.7) with respect to (3.9) does not satisfy the $\beta$-function equations to order $\alpha^{\prime}$ for any choice of the dilaton field.

In order to understand the origin of the problem we go back to the general form of the duality transformation. Since we are interested in conformal invariance, we introduce an arbitrary metric $h_{\alpha \beta}$ on the world-sheet and compute the contribution to the trace anomaly of the auxiliary gauge fields 
$A_{ \pm}^{a}$. This is also justified within string theory where in the first quantized formulation we have to integrate over world-sheet metrics. If for simplicity we work on genus zero surfaces, the most straightforward way to compute the dependence of the effective action on the world-sheet metric is to first parametrize $A_{ \pm}$as:

$$
A_{+}=L^{-1} \partial_{+} L, A_{-}=R^{-1} \partial_{-} R,
$$

for $L, R$ group elements. We can think of $x^{ \pm}$as light-cone variables or as complex coordinates, and they depend on the metric being used. In changing variables from $A_{ \pm}$to $(L, R)$ we encounter jacobians:

$$
\mathcal{D} A_{+} \mathcal{D} A_{-}=\mathcal{D} L \mathcal{D} R \operatorname{det}\left(D_{+}\left(A_{+}\right) D_{-}\left(A_{-}\right)\right)
$$

with $A_{ \pm}$given by (3.10) (we take $A_{ \pm}$as antihermitian matrices). We can write the determinants in (3.11) in terms of a pair of (b,c)-systems $\left(b_{+a}, c^{a}\right)$, $\left(b_{-a}, \tilde{c}^{a}\right) . \quad c, \tilde{c}$ are 0 -forms transforming in the adjoint representation of the group. For arbitrary groups $b_{ \pm a}$ transform in the coadjoint representation. On a general Riemann surface $\Sigma$, if $\mathrm{K}$ is the canonical line bundle, and $\mathrm{E}$ is a vector bundle over $\Sigma, c, \tilde{c}$ are geometrically sections of $\mathrm{E}, c, \tilde{c} \in \Gamma(E)$ for $\mathrm{E}$ the adjoint bundle for a group $\mathrm{G}, b_{+} \in \Gamma\left(K \otimes E^{\sim}\right), b_{-} \in \Gamma\left(\bar{K} \otimes E^{`}\right)$, where $E^{-}$is the dual bundle. The determinants in (3.11) can be exponentiated in terms of the (b,c)- systems with an action:

$$
S\left[b_{ \pm}, c, \tilde{c}\right]=\frac{i}{\pi} \int\left(b_{+} D_{-}(A) c+b_{-} D_{+}(A) \tilde{c}\right),
$$

which is formally conformal invariant. The variation of $\mathrm{S}$ with respect to the metric is given by the energy-momentum tensor $T_{ \pm \pm}$. We can ignore momentarily that $A_{ \pm}$are given by (3.10) and work with arbitrary gauge fields. We can compute the dependence of the effective action for (3.12) on the metric $h_{a b}$ and the gauge field using Feynman graphs, operator product expansions (OPE) or heat kernel methods. Expanding about the flat metric, and using the methods in [9], the first diagrams contributing to the effective action are
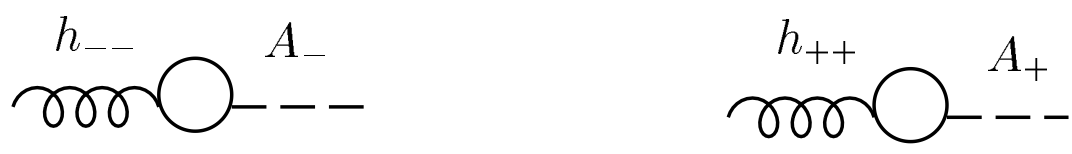
$h_{--}\left(h_{++}\right)$couples to $T_{++}\left(T_{--}\right)$, and $A_{-}\left(A_{+}\right)$to the ghost currents $j_{+}\left(j_{-}\right)$ given by:

$$
\begin{aligned}
& T_{++}=\partial_{+} c^{a} b_{+a}, \quad T_{--}=\partial_{-} \tilde{c}^{a} b_{-a} \\
& j_{+}^{i}=b_{+a}\left(T^{i}\right)_{b}^{a} c^{b}, \quad j_{-}^{i}=b_{-a}\left(T^{i}\right)_{b}^{a} \tilde{c}^{b}
\end{aligned}
$$

If one keeps track of the $i \epsilon$ prescriptions in the propagators appearing in the graphs, the loop integrals are finite, and we can write their contributions to the effective action as:

$$
W^{(2)}=\frac{1}{4 \pi} \operatorname{Tr} T^{a} \int d^{2} p\left(h_{--}(p) \frac{p_{+}^{2}}{p_{-}} A_{-}^{a}(-p)+h_{++}(p) \frac{p_{-}^{2}}{p_{+}} A_{+}^{a}(-p)\right) .
$$

The coefficient of (3.15) and $W^{(2)}$ may also be computed using the OPE:

$$
T(z) j_{a}(w)=\frac{\operatorname{Tr} T_{a}}{(z-w)^{3}}+\frac{1}{(z-w)^{2}} j_{a}(w)+\frac{1}{(z-w)} \partial j_{a}(w) .
$$

As it stands, $W^{(2)}$ has a gravitational anomaly, i.e. the energy-momentum tensor is not conserved. However we can still add local counterterms to (3.15) to recover general coordinate invariance. Since to first order in $h$ the two-dimensional scalar curvature has as Fourier transform:

$$
R(p)=2\left(2 p_{+} p_{-} h_{+-}(p)-p_{+}^{2} h_{--}-p_{-}^{2} h_{++}\right),
$$

if we add the counterterms:

$$
\begin{aligned}
W_{\text {c.t. }} & =\frac{1}{4 \pi} \operatorname{Tr} T_{a} \int A_{-}^{a}(-p)\left(h_{++}(p) p_{-}-2 p_{+} h_{+-}\right) \\
& +\frac{1}{4 \pi} \operatorname{Tr} T_{a} \int A_{+}^{a}(-p)\left(h_{--}(p) p_{+}-2 p_{-} h_{+-}\right),
\end{aligned}
$$

we obtain an effective action

$$
W^{(2)}=\frac{1}{16 \pi} \operatorname{Tr} T_{a} \int R(p) \frac{p_{+} A_{-}^{a}(-p)+p_{-} A_{+}^{a}(-p)}{p_{+} p_{-}},
$$

leading to a conserved energy-momentum tensor, although it contains a trace anomaly which is not proportional to $R(p)$ and therefore it cannot be absorbed in a modification of the dilaton transformation. Varying (3.19) with respect to $h_{+-}$leads to:

$$
<T_{+-}>=\frac{\delta W^{(2)}}{\delta h_{+-}}=\frac{1}{4 \pi} \operatorname{Tr} T_{a}\left(p_{+} A_{-}^{a}(-p)+p_{-} A_{+}^{a}(-p)\right)
$$


which in covariant form it becomes $\sim \operatorname{Tr} T_{a} \nabla^{\alpha} A_{\alpha}^{a}$.

Similarly we can vary the effective action to this order with respect to gauge transformations to evaluate the corresponding gauge anomaly:

$$
\begin{aligned}
& \left(D_{-} \frac{\delta W^{(2)}}{\delta A_{-}^{a}}+D_{+} \frac{\delta W^{(2)}}{\delta A_{+}^{a}}\right) \sim p_{-} \frac{\delta W^{(2)}}{\delta A_{-}^{a}(p)}+p_{+} \frac{\delta W^{(2)}}{\delta A_{+}^{a}(p)} \\
& =p_{-}<j_{a+}(p)>+p_{+}<j_{a-}(p)>=-\frac{1}{8 \pi} \operatorname{Tr} T_{a} R(-p) .
\end{aligned}
$$

This is a different way of writing the third order pole in the OPE (3.16). This result is not surprising when we recall the general theory of b-c systems [15]. The ghost number current $j_{+}=b c$ has an anomaly proportional to $R$ (2d scalar curvature) unless b,c have spin $1 / 2$. In the bosonized form the b-c system is described by an action of the form

$$
\int\left(\partial_{+} \phi \partial_{-} \phi+(2 j-1) R \phi\right) ;
$$

so that $\partial^{\mu} j_{(g h) \mu} \sim R$ as in (3.21) and (3.16), for this reason we cannot gauge the ghost number current unless other compensating fields are included. In our case we can think of the generator whose trace is not zero $\operatorname{Tr} T_{a} \neq 0$ as generating a generalized ghost number current for the auxiliary b-c systems used to construct the measure over $A_{ \pm}$. Since $b_{+}$has spin 1 and c spin 0 , we are certain that the anomaly is there and that there is no consistent gauging, certainly not compatible with conformal invariance. From (3.20) we see that at this order $\left(W^{(2)}\right)$ the trace anomaly is not proportional to $R$, and it therefore cannot be absorbed in a contribution to the dilaton or the effective value of c (the central charge of the Virasoro algebra). The contribution in (3.20) spoils the conformal invariance of the dual theory, and further fields should be required to cancel it. However in that case the resulting theory would not agree with the one obtained through a naive duality transformation.

A third method to obtain the same conclusion as in (3.20) is to use heat kernel methods. Using now complex variables, the problem we are trying to address is the computation of the product of chiral determinants:

$$
\operatorname{det} D_{\bar{z}}(A) \operatorname{det} D_{z}(A)
$$

Each determinant does not make too much sense by itself, not only because it is infinite but because both $D_{z}(A)$ and $D_{\bar{z}}(A)$ map very different spaces 
$D(A): \Gamma[E] \rightarrow \Gamma[K \otimes E]$, and $\bar{D}(A): \Gamma[E] \rightarrow \Gamma[\bar{K} \otimes E]$. There is also a natural pairing between $a \in \Gamma[K \otimes E]$ and $b \in \Gamma\left[\bar{K} \otimes E^{-}\right]$:

$$
\int<a_{z}, b_{\bar{z}}>d z \wedge d \bar{z}
$$

where $<,>$ is the pairing between $E$ and $E^{`}$. To compute determinants we need operators which do not change the space where they act. By looking at the graphical evaluation of the determinants we learn that one is really computing:

$$
\frac{\operatorname{det} \nabla^{z} D_{z}(A) \operatorname{det} \nabla^{\bar{z}} D_{\bar{z}}(A)}{\operatorname{det} \nabla^{z} \nabla_{z}} .
$$

This is manifestly general coordinate invariant, and it can be evaluated with heat-kernel methods. We are in fact interested in the conformal variation of (3.24). Define

$$
H_{+} \equiv \nabla^{z} D_{z}(A), \quad H_{-} \equiv \nabla^{\bar{z}} D_{\bar{z}}(A),
$$

Then under a general variation:

$$
\begin{aligned}
\delta \log \operatorname{det} H_{ \pm} & =-\int_{\epsilon}^{\infty} \frac{d s}{s} \operatorname{Tr} s \delta H_{ \pm} e^{s H_{ \pm}}=-\int_{\epsilon}^{\infty} d s \operatorname{Tr} \delta H_{ \pm} e^{s H_{ \pm}} \\
& =-\int_{\epsilon}^{\infty} d s \frac{\partial}{\partial s} \operatorname{Tr} \delta H_{ \pm} H_{ \pm}^{-1} e^{s H_{ \pm}}=\operatorname{Tr} \delta H_{ \pm} H_{ \pm}^{-1} e^{\epsilon H_{ \pm}(3.26)}
\end{aligned}
$$

Under a conformal transformation $\delta h_{\alpha \beta}=\delta \rho h_{\alpha \beta}$, and

$$
\delta H_{ \pm}=-\delta \rho H_{ \pm},
$$

hence:

$$
\delta_{\rho} \log \operatorname{det} H_{ \pm}=-\operatorname{Tr} \delta \rho e^{\epsilon H_{ \pm}}=-\int d x \delta \rho(x) K_{ \pm}(x, x ; \epsilon),
$$

where $K_{ \pm}(x, y ; \epsilon)$ is the heat kernel of $H_{ \pm}$. To compute the $O\left(\epsilon^{0}\right)$ in $(3.28)$ we can use Gilkey's results [16], or simply take a Fourier transform. To the order at which we are working there is no difference. Hence,

$$
\nabla^{z} D_{z}(A)=\nabla^{z} \nabla_{z}+\left(\nabla^{z} A_{z}\right)+A_{z} \nabla^{z}
$$

and (3.28) becomes:

$$
\delta_{\rho} \log \operatorname{det} H_{+}=-\int d x \delta \rho(x) \int \frac{d^{2} k}{(2 \pi)^{2}} e^{\left[\left(i k^{z}+\nabla^{z}\right)\left(i k_{z}+\nabla_{z}\right)+\nabla^{z} A_{z}+A_{z}\left(\nabla^{z}+i k^{z}\right)\right]} \cdot 1,
$$


rescaling $k \rightarrow \epsilon^{1 / 2} k$, noting that $\int d^{2} k e^{-k^{2}} k^{z} k^{z}=\int d^{2} k e^{-k^{2}} k^{\bar{z}} k^{\bar{z}}=0$, and recalling that $k^{z} k_{z}=\left(k_{x}^{2}+k_{y}^{2}\right) / 2$, because $h_{z \bar{z}}=1 / 2$ for the flat metric, we obtain:

$$
\begin{aligned}
& \delta_{\rho} \log \operatorname{det} H_{+}=-\frac{1}{4 \pi} \int \delta \rho \operatorname{Tr} \nabla^{z} A_{z} \\
& \delta_{\rho} \log \operatorname{det} H_{-}=-\frac{1}{4 \pi} \int \delta \rho \operatorname{Tr} \nabla^{\bar{z}} A_{\bar{z}},
\end{aligned}
$$

as in (3.20), but now the computation is done for the full determinant and not

just to first order in $A$. Hence the diagrammatic and the heat kernel evaluations agree, and we conclude that the condition for the duality transformation to respect conformal invariance is that the generators of the duality group in the adjoint representation should have a vanishing trace. Once again this may happen only for non-semisimple groups, as in the examples discussed at the beginning of this section. Next we will study the non-abelian dual of WZW-models for semisimple groups.

\section{Duality in WZW models}

A very large class of CFT with non-abelian symmetries is embodied in the WZW models [10] for any group $\mathrm{G}$ which we will take to be semisimple, and the GKO-constructions [7, 17].

In this section we present the exact non-abelian duality transformation for a generic WZW-model with group $G$ with respect to the vector action of any of its subgroups. Although we will focus our attention on the non-abelian case, our construction applies as well to abelian, or mixed transformations. Our procedure allows us to determinate the global structure of the nonabelian dual, something which was not yet known. The main result of this section is to show that if we start with a WZW-model with group $\mathrm{G}$ and level $\mathrm{k}$, its global symmetry group is $G_{L} \times G_{R}$, and $G_{D}$ (the diagonal embedding of $\mathrm{G}$ ) is anomaly free. Hence we can select a subgroup $H \subset G_{D}$; and the exact non-abelian dual with respect to $\mathrm{H}$ is the coset model $(G / H)_{k}$ times a WZW-model for $\mathrm{H}$ at level $\mathrm{k}$ (if the Dynkin index of the embedding is one which we take for simplicity; the extension to other cases is straightforward). The basic ingredients in the proof are the use of the Polyakov- Wiegmann (PW) property of the WZW-action together with a special representation of 
the auxiliary field $\chi^{a}$ used in (2.21) to define the starting point of the duality transformation. It is the last step which unravels the global topology hidden in the non-abelian dual.

The WZW-action [10] for a group $\mathrm{G}$ is given by $k S_{0}[g]$,

$$
-4 \pi S_{0}[g]=\int_{M_{2}} d x^{-} d x^{+} \operatorname{Tr}\left(g^{-1} \partial_{+} g g^{-1} \partial_{-} g\right)+\frac{1}{3} \int_{B_{3}, \partial B_{3}=M_{2}} \operatorname{Tr}\left(g^{-1} d g\right)^{3},
$$

where $B_{3}$ is a three-manifold whose boundary is the space-time $M_{2}$ and for compact semisimple groups $\mathrm{k}$ is a positive integer.

A basic property of (4.1) is [18]:

$$
S_{0}\left[g_{1} g_{2}\right]=S_{0}\left[g_{1}\right]+S_{0}\left[g_{2}\right]-\frac{1}{2 \pi} \int \operatorname{Tr}\left(g_{1}^{-1} \partial_{+} g_{1} \partial_{-} g_{2} g_{2}^{-1}\right) .
$$

we next select a subgroup $H \subset G$, and according to the rules of duality we gauge it. The anomaly free condition requires that $H$ should be anomaly free (that (2.20) vanishes), hence we choose the vector action of $h$ on $g$, and at the same time we introduce the gauge fields $A_{ \pm}$(as antihermitian matrices in the Lie algebra of $H$ ). The gauge transformations are:

$$
g \rightarrow h^{-1} g h, A_{ \pm} \rightarrow h^{-1}\left(A_{ \pm}+\partial_{ \pm}\right) h .
$$

The most straightforward way to gauge (4.1) is to use the Noether procedure. After some algebra one obtains [10]

$$
\begin{aligned}
S\left[g, A_{ \pm}\right] & =S_{0}[g]-\frac{1}{2 \pi} \int \operatorname{Tr}\left(A_{+} \partial_{-} g g^{-1}-A_{-} g^{-1} \partial_{+} g\right) \\
& +\frac{1}{2 \pi} \int \operatorname{Tr}\left(A_{+} g A_{-} g^{-1}-A_{+} A_{-}\right) .
\end{aligned}
$$

If we work on spherical world-sheets, we can rewrite $A_{ \pm}$in terms of two group elements:

$$
A_{+}=L^{-1} \partial_{+} L, A_{-}=R^{-1} \partial_{-} R .
$$

Replacing (4.5) in (4.4) and using (4.2) leads to:

$$
S\left[g, A_{ \pm}\right]=S_{0}\left[L g R^{-1}\right]-S_{0}\left[L R^{-1}\right]
$$

Since under gauge transformations:

$$
g \rightarrow h^{-1} g h, L \rightarrow L h, \quad R \rightarrow R h,
$$


we learn that (4.6) is manifestly gauge invariant. In the standard treatment of duality we add the extra term $\chi_{a} F_{+-}^{a}$ and then eliminate the gauge field. However, we find it more convenient to carry the gauge fields along till the end. This will clarify the global properties of the non-abelian dual.

The next step is to transform the auxiliary field term into a more appealing form. We can write $\chi$ and $\mathrm{F}$ as antihermitian matrices valued in the Lie algebra of $H$. With the representation (4.5):

$$
\begin{aligned}
F_{+-} & =\partial_{+} A_{-}-\partial_{-} A_{+}+\left[A_{+}, A_{-}\right] \\
& =\partial_{+}\left(R^{-1} \partial_{-} R\right)-\partial_{-}\left(L^{-1} \partial_{+} L\right)+\left[L^{-1} \partial_{+} L, R^{-1} \partial_{-} R\right] .
\end{aligned}
$$

Defining:

$$
\begin{aligned}
D_{+} f & \equiv \partial_{+} f+\left[L^{-1} \partial_{+} L, f\right] \\
D_{-} f & \equiv \partial_{-} f+\left[R^{-1} \partial_{-} R, f\right],
\end{aligned}
$$

and using the identities:

$$
\begin{aligned}
\partial_{-}\left(L^{-1} \partial_{+} L\right) & =D_{+}\left(L^{-1} \partial_{-} L\right) \\
\partial_{+}\left(R^{-1} \partial_{-} R\right) & =D_{-}\left(R^{-1} \partial_{+} R\right)
\end{aligned}
$$

we can write the gauge field strengh $F_{+-}$as

$$
\begin{aligned}
F_{+-} & =D_{+}\left(R^{-1} \partial_{-} R-L^{-1} \partial_{-} L\right) \\
& =D_{-}\left(R^{-1} \partial_{+} R-L^{-1} \partial_{+} L\right) .
\end{aligned}
$$

Integrating by parts (and up to total derivatives) the auxiliary term becomes:

$$
\begin{aligned}
-\operatorname{Tr} \chi F_{+-} & =\operatorname{Tr} D_{+} \chi\left(R^{-1} \partial_{-} R-L^{-1} \partial_{-} L\right) \\
& =\operatorname{Tr} D_{-} \chi\left(R^{-1} \partial_{+} R-L^{-1} \partial_{+} L\right),
\end{aligned}
$$

Using the identities:

$$
\begin{aligned}
& D_{+} \chi=L^{-1} \partial_{+}\left(L \chi L^{-1}\right) L \\
& D_{-} \chi=R^{-1} \partial_{-}\left(R \chi R^{-1}\right) R
\end{aligned}
$$

and defining the variable

$$
\Sigma=L R^{-1}
$$


we obtain:

$$
\begin{aligned}
\operatorname{Tr} \chi F_{+-} & =-T r \partial_{+}\left(L \chi L^{-1}\right) \Sigma \partial_{-} \Sigma^{-1}+\text { total deriv. } \\
& =\operatorname{Tr} \partial_{-}\left(R \chi R^{-1}\right) \Sigma^{-1} \partial_{+} \Sigma+\text { total deriv. }
\end{aligned}
$$

Collecting our results, the starting point for duality is

$$
\begin{aligned}
& S\left[g, A_{ \pm}\right]+\frac{1}{2 \pi} \int \operatorname{Tr} \chi F_{+-}=k S_{0}\left[L g R^{-1}\right]-k S_{0}[\Sigma] \\
& +\frac{1}{2 \pi} \int \operatorname{Tr} \partial_{-}\left(R \chi R^{-1}\right) \Sigma^{-1} \partial_{+} \Sigma
\end{aligned}
$$

And the quantum theory is defined by integrating over $\left(A_{ \pm}, g, \chi\right)$. Since we find it preferably to work with $L, R$-fields, we need to evaluate the jacobian in the change of variables from $A_{ \pm}$to $(L, R)$. Under infinitesimal changes of $L, R A_{ \pm}$change according to:

$$
\begin{aligned}
& \delta A_{+}=\partial_{+}\left(L^{-1} \delta L\right)+\left[A_{+}, L^{-1} \delta L\right] \\
& \delta A_{-}=\partial_{-}\left(R^{-1} \delta R\right)+\left[A_{-}, R^{-1} \delta R\right]
\end{aligned}
$$

and the change of variables is:

$$
\mathcal{D} A_{+} \mathcal{D} A_{-}=\operatorname{det} D_{+}\left(A_{+}\right) \operatorname{det} D_{-}\left(A_{-}\right) \mathcal{D} L \mathcal{D} R
$$

where the determinants are in the adjoint representation for $A_{ \pm}$as in (4.5). These determinants can be computed by integrating the two-dimensional gauge anomaly [18]. The result is:

$$
\mathcal{D} A_{+} \mathcal{D} A_{-}=\mathcal{D} L \mathcal{D} R e^{-i c_{H} S_{0}[\Sigma]}\left(\operatorname{det} \partial_{+} \operatorname{det} \partial_{-}\right)^{\operatorname{dim} H},
$$

where a counterterm $\int A_{+} A_{-}$has been added to preserve vector gauge invariance. $c_{H}$ is the second Casimir of $H$ in the adjoint representation, and it appears because we write the WZW-action $S_{0}[\Sigma]$ in the fundamental representation. Exponentiating the determinants in (4.19) using two pairs of ghosts $\left(b_{a+}, c^{a}\right),\left(b_{a-}, \tilde{c}^{a}\right)$, we obtain the total action:

$$
\begin{aligned}
& k S_{0}\left[L g R^{-1}\right]-\left(k+c_{H}\right) S_{0}[\Sigma]+\frac{1}{2 \pi} \int \operatorname{Tr} \partial_{-}\left(R \chi R^{-1}\right) \Sigma^{-1} \partial_{+} \Sigma \\
& -\int\left(b_{a+} \partial_{-} c^{a}+b_{a-} \partial_{+} \tilde{c}^{a}\right)
\end{aligned}
$$


integrated over $\left(g, L, R, \chi, b_{ \pm}, c, \tilde{c}\right)$. The first, second and fourth terms in (4.20) look essentially like the functional integral representation for the GKOconstruction of the $(G / H)$ coset conformal field theory [19]. To finally conclude that this is indeed part of the answer we still have to treat the auxiliary field term. However a comparison of (4.20) and (4.1) immediately suggests the following procedure. First introduce the gauge invariant variables

$$
\chi_{R}=R \chi R^{-1}, \chi_{L}=L \chi L^{-1}
$$

next we can think of $\partial_{-} \chi_{R}$ as a gauge connection. On the sphere we can write this gauge connection as a pure gauge field (as in (4.5)):

$$
\partial_{-} \chi_{R}=\left(k+c_{H}\right) \beta^{-1} \partial_{-} \beta,
$$

where $\beta$ takes values on $H$ and the normalization factor $\left(k+c_{H}\right)$ is chosen for convenience. In computing the jacobian factor between $\chi_{R}$ and $\beta$ we proceed in two steps, first we change from $\chi_{R}$ to $\partial_{-} \chi_{R} /\left(k+c_{H}\right)$, this gives a jacobian equal to $\left(\operatorname{det} \partial_{-}\right)^{-\operatorname{dim} H}$; then we compute the change between $\partial_{-} \chi_{R} /\left(k+c_{H}\right)$ and $\beta^{-1} \partial_{-} \beta$. This however is the same computation as the one leading to (4.19) and it is carried out by integrating the anomaly. Thus:

$$
\mathcal{D} \chi=(\mathcal{D} \beta) \exp \left(-i c_{H}\right) S_{0}\left[\beta^{-1}\right]
$$

The $\left(\operatorname{det} \partial_{-}\right)$factors cancel out and there is no need to introduce extra ghosts. Since everything is expressed in terms of gauge invariant variables, we can factor out the volume of the gauge group and integrate only over the combination $\Sigma=L R^{-1}$. With all the previous manipulations we have brought the partition function to the form:

$$
\begin{aligned}
& \int \mathcal{D} g \mathcal{D} \Sigma \mathcal{D} \beta \mathcal{D} b \mathcal{D} c \exp i\left(k S_{0}\left[L g R^{-1}\right]-\left(k+c_{H}\right) S_{0}[\Sigma]\right. \\
& \left.-\frac{k+c_{H}}{2 \pi} \int \operatorname{Tr} \beta^{-1} \partial_{-} \beta \Sigma^{-1} \partial_{+} \Sigma-c_{H} S_{0}\left[\beta^{-1}\right]+\text { ghosts }\right) .
\end{aligned}
$$

Using (4.2) once again leads to:

$$
\begin{aligned}
& \int \mathcal{D} g \mathcal{D} \Sigma \mathcal{D} \beta \mathcal{D} b \mathcal{D} c \exp i\left(k S_{0}\left[L g R^{-1}\right]-\left(k+c_{H}\right) S_{0}\left[\Sigma \beta^{-1}\right]+k S_{0}\left[\beta^{-1}\right]\right. \\
& \left.-\int\left(b_{a+} \partial_{-} c^{a}+b_{a-} \partial_{+} \tilde{c}^{a}\right)\right) .
\end{aligned}
$$


Now we can make the change of variables $g \rightarrow L g R^{-1}, \Sigma \rightarrow \Sigma \beta^{-1}$ with unit jacobian. This leads to an action:

$$
k S_{0}[g]-\left(k+c_{H}\right) S_{0}[\Sigma]-\int\left(b_{a+} \partial_{-} c^{a}+b_{a-} \partial_{+} \tilde{c}^{a}\right)+k S_{0}\left[\beta^{-1}\right] .
$$

Although the action looks decoupled, $(g, \Sigma, b, c)$ are coupled through the physical state condition. Since we have gauged $H$, there is BRST charge receiving contributions from $g, \Sigma, b, c$ given by [19]:

$$
Q=\oint c^{a}\left(J_{+a}(g)+J_{+a}(\Sigma)-\frac{1}{2} c^{m} f_{a m}^{n} b_{+n}\right),
$$

where $J_{+a}(g)$ are the left-handed $H$-currents from $k S_{0}[g], J_{+a}(\Sigma)$ the left- $H$ currents from $-\left(k+c_{H}\right) S_{0}[\Sigma]$ and the third contribution comes from the ghosts. It is easy to check that

$$
\left\{Q, b_{+a}\right\}=J_{+a}(g)+J_{+a}(\Sigma)+J_{+a}^{\text {ghost }}
$$

then $Q^{2}=0$ as long as the total current has vanishing central charge. This is indeed the case because $J_{+}(g)$ has central charge $\mathrm{k}, J_{+}(\Sigma)$ has $-\left(k+c_{H}\right)$ and $J_{+}^{g h}+c_{H}$. Hence the first three terms in (4.26) together with the physical state condition $Q \mid$ phys $>=0$ provide a functional integral representation of the coset conformal field theory $G / H$. Since the energy-momentum tensor associated to WZW-models is of Sugawara form, the total central charge of the Virasoro algebra coming from the first three terms is easily computed once we use the relation noted in Karabali et al [19]:

$$
c\left(H,-k-c_{H}\right)-2 \operatorname{dim} H=-c(H, k) .
$$

The contribution $-2 \operatorname{dim} H$ comes from the b-c system, and as mentioned before we are assuming that the embedding $H \subset G_{D}$ has Dynkin index equal to 1 , otherwise there are obvious changes in the normalization factors for the WZW-models for the subgroup $\mathrm{H}$ with respect to that of $\mathrm{G}$ (and this also affects $S_{0}\left[\beta^{-1}\right]$ ). Hence although we have not explicitly eliminated the gauge field variables $\Sigma$ in the classical Lagrangian (4.26), it is clear that (4.26) describes the non-abelian dual. What is more important is that (4.26) brings out the global topology of the auxiliary field $\chi$ once it is represented in terms of $\beta$. Although the relation between $\chi_{R}$ and $\beta$ is non-local, the 
Lagrangians (4.26), (4.24) are local and we can study their quantum theory with standard methods.

If we want to explicitly eliminate the field $\Sigma$ altogether, we may proceed as follows. Since $H \subset G_{D}$, we may orthogonally decompose the Lie algebra of $\mathrm{G} \underline{g}=\underline{h} \oplus \underline{k}$, where $[\underline{h}, \underline{k}] \subset \underline{k}$. Then the element $g \in G$ can be written as $g=l h$, with $h \in H$, and $l^{-1} d l \in \underline{k}$. Since the decomposition $\underline{h} \oplus \underline{k}$ is orthogonal,

$$
S_{0}[l h]=S_{0}[l]+S_{0}[h]
$$

with the last term in (4.2) equal to zero due to orthogonality. Hence the action takes the form

$$
S_{\text {dual }}=k S_{0}[l]+k S_{0}\left[\beta^{-1}\right]+\left(k S_{0}[h]-\left(k+c_{H}\right) S_{0}[\Sigma]+\text { ghost }\right) .
$$

The terms between parenthesis represent a CFT with $c=0$, and the BRST conditions only involve the $H$-valued fields $h, \Sigma$ and the ghosts $b_{ \pm}, c, \tilde{c}$. Hence these terms represent an $(H / H)$ theory with $c=0$ whose Hilbert space is reduced to the identity operator. Therefore we can set the integral over $\left(h, \Sigma, b_{ \pm}, c, \tilde{c}\right)$ (taking into account the BRST condition) to 1 , and write the dual action explicitly in terms of the coset variables $l$ together with a level $\mathrm{k}$ WZW-model for the $H$-valued auxiliary field. This completes the proof of the main statement at the beginning of the section. We now present some consequences and remarks concerning the results presented.

It should be clear from the previous arguments that if we consider a coset CFT $G / H$ such that the centralizer $H^{\prime}$ of $H$ in $G$ is not trivial, we can perform duality with respect to $H^{\prime}$, and the result will be (4.26) with two extra terms, $-\left(k+c_{H^{\prime}}\right) S\left[\Sigma^{\prime}\right]+S_{\mathrm{gh}}\left[b^{\prime}, c^{\prime}\right]$ associated to $H^{\prime}$.

Another simple consequence of our arguments is that they can readily be specialized to the case when $H$ is abelian. In this case the dual CFT is given in terms of $G / H$ parafermions and a collection of free fields living on the torus defined by $H$, as one expect from standard arguments of abelian duality (see for example the review by E. Kiritsis in [2]).

We remark further that we have not written an explicit form for the metric induced in $G / H$ by our construction. This is reasonable because we have carried out an exact quantum treatment. In the limit as $k \rightarrow \infty$ the leading form of the metric is given by the one obtained from the left hand side of (4.16) after eliminating the gauge fields $A_{ \pm}$and then fixing the gauge. However we expect corrections to all orders in $1 / \mathrm{k}$, as exemplified 
by the corrections to the two-dimensional String Black Hole [20] described in [21]. There are in principle methods to compute systematically the $1 / \mathrm{k}$ corrections to the classical metric [22]. The advantage of working without explicitly using the metric is that the classical metric for the dual theory with respect to $H \subset G$ is quite singular, there are fixed submanifolds of $G$ under the action of $H$, thus leading to nasty singularities in the naive metric; and this makes it nearly hopeless to obtain (4.31) in the standard $\sigma$-model approach.

One important point in our derivation of (4.31) was the relative normalization between $\chi_{R}$ and $\beta$ (the factor $k+c_{H}$ ). In the original approach, the normalization of $\chi$ was not fixed. In the abelian case the range of the auxiliary field $\chi$ (which for compact abelian $H$ is a torus) is determined by requiring the absence of modular anomalies for world-sheets with genus higher than zero (see for instance [6], [4] and [23]). This related the size of the orbits of the isometry group with the size of the dual torus where the auxiliary field takes its values. More explicitly this is a consequence of requiring that the topological term in the second line in (2.10) should have integral values upon integration over the world-sheet. The analysis of modular invariance in genus one is more complicated for $(4.26,31)$, but it is certainly necessary to determine the detailed operator mapping. The partition function at genus one of the original WZW-model is not a product of any modular invariant partition function for the $G / H$ coset theory and one of the $H$ WZW-model. The Kac-Moody characters of the $G_{k}$ WZW-model have a well-defined decomposition in terms of products of $G / H$ and $H_{k}$ characters. We have seen that the relative normalization in (4.22) is justified by the matching of the Virasoro central extension for the $G_{k}$ model and its dual. Further justification comes from the analysis on the torus concerning modular invariance. Now it is not sufficient to write $A_{+}=L^{-1} \partial_{+} L, A_{-}=R^{-1} \partial_{-} R$, but one has to include the contribution from flat connections. The arguments follow closely the evaluation of $G / H$ character in the work of Gawedzki and Kupiainen [19]. The details will be presented elsewhere.

Summarizing the field $\beta$ related to $\chi_{R}$ by (4.22) is indeed valued in the group manifold $H$; and this determines the global properties of the nonabelian dual.

It is worth noting to conclude this section that the fields $\chi_{L}, \chi_{R}$ resemble the non-local fields used in the standard Kramers-Wannier duality transformation [1]. For instance $\chi_{R}$ is constructed by multiplying $\chi(x)$ by the Wilson 
line for $A_{-}$from $-\infty$ to x on the left, and by the $A_{-}$Wilson line from x to $\infty$ on the right. From this point of view it is also satisfying that the physical operators in the dual theory $(4.26,31)$ contain contributions from $\beta$ which are an analogue of the disorder operator in the Ising model.

\section{General $\sigma$-models with chiral currents}

Using the results of Hull and Spence [12] quoted in section 2 we want to study the general case of a $\sigma$ - model where there are left and right-chiral currents. We will also present a "heterotic" generalization of the treatment of WZW-models carried out in the previous section. The aim of this section is to prove that for a general $\sigma$-model with $G_{L} \otimes G_{R}$ isometry group acting on a manifold $\mathrm{M}$, the non-abelian dual with respect to $G_{D} \subset G_{L} \otimes G_{R}$ is again a generalized GKO construction (M/G) times a WZW-model for G. To make the arguments as clear as possible, consider first a model with only left chiral currents: $\partial_{-} J_{+a}=0$. From the equations (2.11-21), we have $v_{a i}=-\xi_{a i}$, so that the action becomes:

$$
4 \pi S\left[\phi, A_{-}\right]=\int_{M_{2}} g_{i j} \partial_{+} \phi^{i} \partial_{-} \phi^{j}+\frac{1}{3} \int_{B_{3}, \partial B_{3}=M_{2}} H_{i j k} d \phi^{i} d \phi^{j} d \phi^{k}-2 \int A_{-}^{a} \xi_{a i}^{L} \partial_{+} \phi^{i}
$$

This model has an anomalous variation:

$$
4 \pi \delta S[\phi, A]=-2 \int A_{-}^{a} \xi_{a} \cdot \xi_{b} \partial_{+} \epsilon^{b} .
$$

$\xi_{a} \cdot \xi_{b}$ are constants independent of $\phi$ (see the proof after equation (2.20) when $\left.v_{a i}= \pm \xi_{a i}\right)$. Note that only $A_{-}^{a}$ appears in (5.1). Hence we can take $A_{-}=L^{-1} \partial_{-} L$, and in fact define also $A_{+}=L^{-1} \partial_{+} L$, as though A were pure gauge. By adding and substracting appropriate terms in (5.1), we obtain:

$$
\begin{aligned}
4 \pi S[\phi, A] & =\int g_{i j} D_{+} \phi^{i} D_{-} \phi^{j}+\frac{1}{3} \int_{B_{3}} H_{i j k} d \phi^{i} d \phi^{j} d \phi^{k} \\
& +\int\left(A_{+}^{a} \xi_{a i}^{L} \partial_{-} \phi^{i}-A_{-}^{a} \xi_{a i}^{L} \partial_{+} \phi^{i}\right)-\int \xi_{a} \cdot \xi_{b} A_{+}^{a} A_{-}^{b}
\end{aligned}
$$

where $D_{ \pm} \phi^{i}=\partial_{ \pm} \phi^{i}-\xi_{a}^{i} A_{ \pm}^{a}$. Next, we can use the identity (2.23) [12] to rewrite (5.3) as:

$$
4 \pi S[\phi, A]=\int g_{i j} D_{+} \phi^{i} D_{-} \phi^{j}+\frac{1}{3} \int_{B_{3}} H_{i j k} D \phi^{i} D \phi^{j} D \phi^{k}
$$




$$
\text { - } k \int_{B_{3}}\left(A^{a} d A^{a}+\frac{1}{3} f_{a b c} A^{a} A^{b} A^{c}\right)-k \int \delta_{a b} A_{+}^{a} A_{-}^{b},
$$

since we can take the gauge one-form $\mathrm{A}$ as pure gauge $A=L^{-1} d L$, the curvature term $F^{a}$ in (2.23) drops out. Also using the fact that $\xi_{a} \cdot \xi_{b}$ is a constant symmetric metric, we have chosen a basis to diagonalize it. The normalization factor $\mathrm{k}$ depends on the normalization of the three form $\mathrm{H}$, and it is an integer for compact semisimple groups. For $A=L^{-1} d L$, the Chern-Simons term becomes:

$$
k \int\left(A^{a} d A^{a}+\frac{1}{3} f_{a b c} A^{a} A^{b} A^{c}\right)=-\frac{k}{3} \int_{B_{3}} \operatorname{Tr}\left(L^{-1} d L\right)^{3},
$$

and consequently the last two terms in (5.4) produce a WZW-action for G at level $\mathrm{k}$. The first two terms can be written now in terms of the original theory. The group $\mathrm{G}$ acts on the manifold through some functions $f: G \times M \rightarrow M$, $\phi \rightarrow f(\phi ; L)$. Using the invariance of $g_{i j}$ and $H_{i j k}$ under the action of the group, we can make the change of variables $\phi^{i} \rightarrow \phi^{\prime}{ }^{i}=f^{i}(\phi ; L(x))$, with this change of variables the first two terms in (5.4) become the original ungauged action written in terms of $\phi^{\prime}$. Hence:

$$
S[\phi, A]=\frac{1}{4 \pi} \int g_{i j}\left(\phi^{\prime}\right) \partial_{+} \phi^{\prime}{ }^{i} \partial_{-} \phi^{\prime} j+\frac{1}{12 \pi} \int H_{i j k}\left(\phi^{\prime}\right) d \phi^{\prime i} d \phi^{\prime j} d \phi^{\prime k}-k S_{0}^{\mathrm{WZW}}[L]
$$

Thus we decouple the $\sigma$-model variables and the gauge variables. The model (5.6) is anomalous, and therefore the standard duality transformation does not work. We can nevertheless generalize the duality transformation to this case by analogy with the similar situation in the abelian case (see first entry in [4]). We cancel the anomaly in (5.6) by giving special gauge transformation properties to the auxiliary field $\beta$ (see (4.16)-(4.22)). If we want to perform duality with respect to the left-acting group $G_{L}$, we only need to integrate over $A_{-}$. Since the field $A_{+}$is not present, we write the auxiliary field term in the form (4.20)

$$
\frac{1}{2 \pi} \int \operatorname{Tr} \partial_{-} \chi L^{-1} \partial_{+} L
$$

Making the change of variables $A_{-} \rightarrow L$ and as in (4.22) $\left(k+c_{G}\right) \partial_{+} \chi=$ $-\beta^{-1} \partial_{+} \beta$, together with the Polyakov-Wiegmann property (4.2), we arrive at

$$
\frac{1}{4 \pi} \int g_{i j}\left(\phi^{\prime}\right) \partial_{+} \phi^{\prime} \partial_{-} \phi^{\prime j}+\frac{1}{12 \pi} \int H_{i j k}\left(\phi^{\prime}\right) d \phi^{\prime i} d \phi^{\prime j} d \phi^{\prime k}
$$




$$
-\left(k+c_{G}\right) S_{0}^{\mathrm{WZW}}\left[L \beta^{-1}\right]+k S_{0}\left[\beta^{-1}\right]+\operatorname{ghosts}(b, c)
$$

This is again interpreted as a coset construction $\left(M / G_{L}\right)$ times a WZWmodel for $G_{L}$ at level k. Note that the term (5.7) (if written in terms of $\beta$ ) is precisely what is needed to cancel the gauge anomaly in (5.6). As a further remark, note that the original gauged action (5.3) together with (5.7) are equivalent to the ungauged theory (5.1) with $A_{-}=0$. This is because the equations of motion derived from (5.7) imply that we can choose the gauge transformation $\mathrm{L}$ as a function of $x^{+}$only. But since the original theory has left-chiral invariance, a simple change of variables recovers the starting action. This construction can be generalized to perform non-abelian duality with respect to separate groups on the left and on the right, provided we include two auxiliary fields $\beta_{L}, \beta_{R}$ needed to cancel the anomaly. This should have interesting consequences for heterotic $\sigma$-models, which are currently under investigation.

The case with left- and right-handed currents parallels the previous arguments and those of section 4 . Once again it is crucial to use the identity (2.23). Imagine we start with a general WZW-model

$$
4 \pi S_{0}[\phi]=\int g_{i j}(\phi) \partial_{+} \phi^{i} \partial_{-} \phi^{j}+\frac{1}{3} \int H_{i j k} d \phi^{i} d \phi^{j} d \phi^{k},
$$

with left-handed and right-handed isometries. Then, under

$$
\delta \phi^{i}=\epsilon_{L}^{a}(x) \xi_{L a}^{i}-\epsilon_{R}^{a}(x) \xi_{R a}^{i},
$$

(5.9) changes to

$$
4 \pi \delta S_{0}[\phi]=2 \int\left(\xi_{a L i} \partial_{+} \phi^{i} \partial_{-} \epsilon_{L}^{a}-\xi_{a R i} \partial_{-} \phi^{i} \partial_{+} \epsilon_{R}^{a}\right)
$$

The left- and right-Killing vectors satisfy the commutation relations:

$$
\begin{aligned}
& {\left[\xi_{L a}, \xi_{L b}\right]=f_{a b}^{c} \xi_{L c}} \\
& {\left[\xi_{R a}, \xi_{R b}\right]=-f_{a b}^{c} \xi_{R c}} \\
& {\left[\xi_{L a}, \xi_{R b}\right]=0}
\end{aligned}
$$

To cancel (5.11) we introduce:

$$
4 \pi S_{1}[\phi, A]=-2 \int\left(\xi_{a L i} \partial_{+} \phi^{i} A_{L-}^{a}-\xi_{a R i} \partial_{-} \phi^{i} A_{R+}^{a}\right)
$$


One can continue like this until we obtain an action whose variation is independent of $\phi$. If we continue gauging the left and right isometries, we of course obtain an anomalous theory. Then we have two options, one is to follow the heterotic path explained before, and the second is to restrict the gauging to the diagonal, anomaly free subgroup. We will follow the latter. Since

$$
\delta A_{L, R}^{a}=\partial \epsilon_{L, R}^{a}+f_{b c}^{a} A_{L, R}^{b} \epsilon_{L, R}^{c}
$$

take now:

$$
\epsilon_{L}^{a}=\epsilon_{R}^{a}=\epsilon^{a}
$$

Choosing a basis such that:

$$
\xi_{L a} \cdot \xi_{L b}=\xi_{R a} \cdot \xi_{R b}=k \delta_{a b},
$$

we find that the following action is vector-gauge invariant:

$$
\begin{aligned}
4 \pi S\left[\phi, A_{L}, A_{R}\right] & =\int g_{i j}(\phi) \partial_{+} \phi^{i} \partial_{-} \phi^{j}+\frac{1}{3} \int H_{i j k} d \phi^{i} d \phi^{j} d \phi^{k} \\
& -2 \int\left(\xi_{a L i} \partial_{+} \phi^{i} A_{L-}^{a}-\xi_{a R i} \partial_{-} \phi^{i} A_{R+}^{a}\right) \\
& +2 \int\left(\xi_{a L} \cdot \xi_{b L}-\xi_{a L} \cdot \xi_{b R}\right) A_{L-}^{a} A_{R+}^{b}
\end{aligned}
$$

One important property of (5.17) is that $A_{L}^{a}\left(A_{R}^{a}\right)$ only appears in the Lagrangian through its $A_{L-}^{a}\left(A_{R+}^{a}\right)$ component. Thus we can take $A_{L}=L^{-1} d L$, $A_{R}=R^{-1} d R$, although only the - and + respectively components do appear. This is useful when we use the identity (2.11) to write the final form of the action. Since the full covariant derivatives are:

$$
D_{ \pm} \phi^{i}=\partial_{ \pm} \phi^{i}-\xi_{L a}^{i} A_{L \pm}^{a}+\xi_{R a}^{i} A_{R \pm}^{a},
$$

as we did in the chiral case we can add and substract some terms to (5.17) to complete the covariant derivatives. The result is:

$$
\begin{aligned}
& 4 \pi S\left[\phi, A_{L}, A_{R}\right]=\int g_{i j} D_{+} \phi^{i} D_{-} \phi^{j}+\frac{1}{3} \int H_{i j k} d \phi^{i} d \phi^{j} d \phi^{k} \\
& +\int\left(\xi_{a L i} d \phi^{i} \wedge A_{L}^{a}+\xi_{a R i} d \phi^{i} \wedge A_{R}^{a}\right)+\int \xi_{a L} \cdot \xi_{b R}\left(A_{L+}^{a} A_{R-}^{b}-A_{L-}^{a} A_{R+}^{b}\right) \\
& -\int\left(\xi_{L a} \cdot \xi_{L b} A_{L+}^{a} A_{L-}^{b}+\xi_{R a} \cdot \xi_{R b} A_{R+}^{a} A_{R-}^{b}\right)+2 \int \xi_{a L} \cdot \xi_{b L} A_{L-}^{a} A_{R+}^{b} .(5.19)
\end{aligned}
$$


The first four terms on the right-hand side of (5.19) can be combined using (2.23) to produce $\frac{1}{3} H_{i j k} D \phi^{i} D \phi^{j} D \phi^{k}$ on the three-dimensional integral $\left(D \phi^{i}=d \phi^{i}-\xi_{L a}^{i} A_{L}^{a}+\xi_{R a}^{i} A_{R}^{a}\right)$, together with the Chern-Simons terms for $A_{L}$ and $A_{R}$. Since $A_{L}=L^{-1} d L, A_{R}=R^{-1} d R$, these together with the last two terms in (5.19) reconstruct the WZW-action for $\mathrm{G}$ at level-k, where $\mathrm{k}$ is the factor in (5.16). Summarizing we obtain:

$$
4 \pi S\left[\phi, A_{L}, A_{R}\right]=\int g_{i j} D_{+} \phi^{i} D_{-} \phi^{j}+\frac{1}{3} \int H_{i j k} D \phi^{i} D \phi^{j} D \phi^{k}-k S_{0}^{\mathrm{WZW}}\left[L R^{-1}\right]
$$

Now since the group $G_{L} \otimes G_{R}$ acts on M, we can make again the change of variables $\phi^{i} \rightarrow \phi^{\prime}{ }^{i}=f^{i}(\phi ; L, R)$, and the invariance of $g_{i j}, H_{i j k}$ imply that

$$
4 \pi S\left[\phi, A_{L}, A_{R}\right]=4 \pi S\left[\phi^{\prime}, 0,0\right]-k S_{0}^{\mathrm{WZW}}\left[L R^{-1}\right],
$$

where $S_{0}^{\mathrm{WZW}}\left[L R^{-1}\right]$ is the WZW-action (4.1). Once $\phi^{\prime}, L, R$ are decoupled, the rest of the argument is a rerun of the WZW case considered in the previous section and we omit it. This proves the statement at the beginning of this section.

In the case when the isometries are not chiral (i.e. when we have the gauge Lagrangian $(2.18,23)$ ), the treatment for the measure for $A_{+}, A_{-}$and the change of variables from $\chi$ to $\beta$ can be done along the same lines; however one does not have a neat decoupling between the $\phi^{i}$ variables and $(L, R, \beta)$, for this reason it is not clear what is the correct normalization factor $\lambda \partial_{-} \chi=$ $\beta^{-1} \partial_{-} \beta$. As in the WZW case $\lambda$ should be determined in order to match the central charge of the initial and final conformal field theories, but we do not yet have a general procedure to compute $\lambda$ explicitly. Note that $\lambda$ is crucial in order to determine the global topology of the auxiliary variable $\beta$ in the dual theory. Thus the exact form of the transformation in the general case when there are no chiral currents remains open. One extreme case corresponds to $\sigma$-models with $H=0$. There we have to study each model separately.

In the next section we analyze in detail two simple examples based on the Eguchi-Hanson and Taub-Nut gravitational instantons [11] which have $S U(2)_{L} \times U(1)_{R}$ as isometry groups. 


\section{Duals of gravitational instantons}

Some interesting examples of non-abelian duality transformations have been already worked out in previous papers [4],[5], including WZW-models with $G=S U(2)$ (or $S L(2, R)$ ) with respect to its own left action (getting in the latter case a three-dimensional black hole).

We would like to examine here another interesting class of examples, namely the asymptotically locally Euclidean self-dual solutions to Euclidean gravity (gravitational instantons). All known $S U(2)$-symmetric solutions [27] fall within the framework of a Bianchi IX metric:

$$
d s^{2}=f^{2}(r) d r^{2}+\sum_{i=1}^{3} a_{l}^{2}(r)\left(\operatorname{Tr} \frac{i \sigma_{l}}{2}\left(g^{-1} d g\right)\right)^{2}
$$

The elements of $S U(2)$ are here parametrized by their Euler angles,

$$
g=e^{i \frac{\phi}{2} \sigma_{3}} e^{i \frac{\theta}{2} \sigma_{2}} e^{i \frac{\psi}{2} \sigma_{3}}
$$

where $0 \leq \theta \leq \pi, 0 \leq \phi \leq 2 \pi, 0 \leq \psi \leq 4 \pi$. In this form all these metrics are invariant under the left action of $S U(2): g \rightarrow h g$ (that is, the induced action on the Euler angles parametrizing a sphere $\left.S^{3}\right)$.

The celebrated Eguchi-Hanson (EH) metric is a particular case corresponding to

$$
f^{2}=\frac{1}{1-\left(\frac{a}{r}\right)^{4}}, \quad a_{1}^{2}=a_{2}^{2}=r^{2}, \quad a_{3}^{2}=r^{2}\left(1-\left(\frac{a}{r}\right)^{4}\right)
$$

and the Euclidean Taub-Nut (ETN) solution to

$$
f^{2}=\frac{1}{4} \frac{r+m}{r-m}, a_{1}^{2}=a_{2}^{2}=r^{2}-m^{2}, \quad a_{3}^{2}=m^{2} \frac{r-m}{r+m}
$$

We can treat both cases (as well as the, less interesting from our point of view, Fubini-Study metric on $P_{2}(\mathcal{C})$ ) simultaneously, by considering the twodimensional $\sigma$-model corresponding to the general Bianchi IX ansatz, namely:

$$
S=\frac{1}{2 \pi} \int\left(f^{2}(r) \partial r \bar{\partial} r+\sum_{l=1}^{3} a_{l}^{2}\left(\operatorname{Tr} \frac{i \sigma_{l}}{2} g^{-1} \partial g\right)\left(\operatorname{Tr} \frac{i \sigma_{l}}{2} g^{-1} \bar{\partial} g\right)\right)
$$


We would like to consider the left action of the full $S U(2)$ group (with respect to which the two-dimensional field $r$ is inert), and work in the unitary gauge, $g=1$. This yields after some standard manipulations:

$$
\tilde{S}=\frac{1}{2 \pi} \int f^{2}(r) \partial r \bar{\partial} r+\frac{4}{\Delta(r, \chi)}\left(16 \chi_{i} \chi_{j}+4 \epsilon_{i j k} \chi_{k} a_{k}^{2}+\delta_{i j} \frac{a_{1}^{2} a_{2}^{2} a_{3}^{2}}{a_{i}^{2}}\right) \partial \chi_{i} \bar{\partial} \chi_{j}
$$

where $\Delta(r, \chi) \equiv a_{1}^{2} a_{2}^{2} a_{3}^{2}+16 \sum a_{i}^{2} \chi_{i}^{2}$ and a dual dilaton given by [3]

$$
\tilde{\phi}=\log \frac{\Delta}{64}
$$

Please note the similarities of these formulas with (7.8) of first reference in [4]. We also find, like we did there, that there is both a dual metric and a dual torsion. To be specific, the dual to the Eguchi-Hanson solution is:

$$
\begin{aligned}
d \tilde{s}_{E H}^{2} & =\frac{4 r^{4}}{r^{4}-a^{4}} d r^{2}+\frac{r^{2}}{\left(r^{4}-a^{4}\right)\left(r^{4}+\xi^{2}\right)+r^{4} \rho^{2}}\left(\left(\rho^{2}+r^{4}-a^{4}\right) d \rho^{2}\right. \\
& \left.+\left(r^{4}+\xi^{2}\right) d \xi^{2}+2 \rho \xi d \rho d \xi+\left(r^{4}-a^{4}\right) \rho^{2} d \phi^{2}\right)
\end{aligned}
$$

where we have introduced cylindrical coordinates in the space of the Lagrange multipliers

$$
\chi_{1}=\rho \cos \phi, \quad \chi_{2}=\rho \sin \phi, \quad \chi_{3}=\xi
$$

and rescaled $r$ and $a$ by a factor of 2 .

The dilaton is given by

$$
\tilde{\phi}_{E H}=\log \left(\frac{\left(r^{4}-a^{4}\right)\left(r^{4}+\xi^{2}\right)+r^{4} \rho^{2}}{r^{2}}\right)
$$

and the torsion is

$$
\tilde{b}_{E H}=\frac{\rho}{\left(r^{4}-a^{4}\right)\left(r^{4}+\xi^{2}\right)+r^{4} \rho^{2}}\left(\xi\left(r^{4}-a^{4}\right) d \rho \wedge d \phi+r^{4} \rho d \phi \wedge d \xi\right)
$$

The dual metric is asymptotically flat as $r \rightarrow \infty$ since it can be checked that the Riemann tensor vanishes in this limit.

It (like the original EH metric) also has an apparent singularity at $r=$ $a$, which can also be resolved as a bolt (without any need to change the periodicity of our coordinates). The dual metric is not self-dual (it is not Ricci-flat); an amusing point is, however, that the corresponding string metric 
$d \tilde{s}_{S E H}^{2} \equiv e^{\tilde{\phi}} d \tilde{s}_{E H}^{2}$ is not only self-dual, but absolutely flat; the corresponding Riemann tensor is zero.

The other interesting example is the ETN metric, whose dual reads

$$
\begin{aligned}
& d \tilde{s}_{E T N}^{2}=\frac{r+m}{r-m} d r^{2}+\frac{1}{\Sigma(r, \rho, \xi)}\left(\left(\rho^{2}+4 m^{2}(r-m)^{2}\right) d \rho^{2}\right. \\
& \left.+\left(\xi^{2}+\left(r^{2}-m^{2}\right)^{2}\right) d \xi^{2}+2 \rho \xi d \rho d \xi+4 m^{2}(r-m)^{2} \rho^{2} d \phi^{2}\right)
\end{aligned}
$$

where $\Sigma(r, \rho, \xi) \equiv \frac{r-m}{r+m}\left[4 m^{2}\left(\xi^{2}+\left(r^{2}-m^{2}\right)^{2}\right)+\rho^{2}(r+m)^{2}\right]$.

The dilaton is given by $\tilde{\phi}_{E T N}=\log \Sigma$ and the torsion reads

$$
\tilde{b}_{E T N}=\frac{\rho(r-m)}{r+m} \frac{1}{\sum(r, \rho, \xi)}\left(4 \xi m^{2} d \rho \wedge d \phi+\rho(r+m)^{2} d \phi \wedge d \xi\right)
$$

The scalar curvature tends to a positive constant $\left(5 / 2 \mathrm{~m}^{2}\right)$ asymptotically as $r \rightarrow \infty$. In this case, however, the singularity at $r=m$ is not apparent, but a true curvature singularity $\left(\tilde{R} \sim \frac{3}{4 m} \frac{1}{r-m}\right)$. The different behavior in this case as compared with $d \tilde{s}_{E H}^{2}$ stems from the fact that the norms of the killing vectors vanish when $r=m$, which means that the left action of $S U(2)$ has fixed points in the four-dimensional manifold precisely at $r=m$.

As a matter of principle, it is interesting to know whether there are self-dual solutions to the beta function equations of conformal invariance of Callan et al, with a non-trivial dilaton field. In the strictest sense of the word, the only possibility allowed is a Ricci-flat spacetime. Solutions of the beta-function equations with these characteristics can be obtained with zero torsion, and linear dilaton field.

There is a possible avenue for a further generalization. It is well known that one can recover all the beta-function equations (with frozen dilaton) by considering the Ricci flatness condition, but with respect to the generalized connection $\omega_{b}^{a}=\gamma_{b}^{a}+h_{b}^{a}$, where $\gamma$ is the usual Levi-civita connection, and $h$ is the connection giving rise to the torsion $H$. (The Ricci tensor is now non-symmetric, so both the symmetric and the antisymmetric parts must be zero; this is why we get two equations from a single condition). A natural conjecture is that there might exist a rescaling of the vierbein (and perhaps of the torsion as well) such that the Ricci flatness condition of the corresponding rescaled torsion conveys the beta function equations, with a dynamical dilaton.

An easy calculation, however, shows this not to be the case, because one can not get rid of all terms containing derivatives of the dilaton field. 


\section{Conclusions and outlook}

In this paper we have presented a general analysis of non-abelian duality. We have shown that there is a potential obstruction to conformal invariance for non-semisimple groups. This obstruction is embodied in a mixed gauge and gravitational anomaly appearing in the measure of integration over the gauge fields. This solves the puzzle raised in [8] which was one of the motivations for our work. We then presented the exact form of the non-abelian duality transformation for WZW-models with respect to any of its anomaly free subgroups, and we extended the construction to coset conformal field theories and to conformal theories on manifolds with left and right chiral currents. Our treatment of the auxiliary field allowed us also to define duality with respect to anomalous subgroups by using the auxiliary field to cancel the anomaly. This opens the way to perform duality with respect to independent left and right groups which should have interesting applications in heterotic $\sigma$-models. The general case without chiral currents is unfortunately still out of reach with our methods. However we presented some moderately interesting examples of non-abelian duality for four-dimensional gravitational instantons. There are a number of open problems that we would like to list here.

1. It could be interesting to work out the general structure of the dual theory when there are no chiral currents. We are still far from a satisfactory understanding of the general case.

2. We still do not have a clear picture of whether non-abelian duality can be "inverted" as in the abelian case. Although in the WZW or GKOmodels we have characterized completely the dual theory, it is only a posteriori that we can deduce the starting theory.

3. The fact that we can explicitly characterize the non-abelian dual of a large class of theories makes us suspect that there should be an analog of this duality in lattice field theories. However the explicit construction, and how the absence of Pontrjagin duality for non-abelian groups is circumvented remains to be explored.

4. The explicit operator mapping is still to be developed fully. However we feel that the methods presented here should help in completing this task. 
5. The heterotic version of duality presented in section five remains to be explored.

6. Dijkgraaf and Witten [25] showed in the context of Chern-Simons theory how to gauge discrete subgroups in the continuum. Given the close relation between Chern-Simons theory and WZW-models, it seems reasonable to expect that a combination of the methods presented in our paper together with those in [25] should allow us to define duality transformations with respect to discrete isometry groups. If this is possible, a large and interesting class of examples where one could try discrete duality is in Calabi-Yau manifolds; of importance in String Theory compactifications [26].

7. The extension of our construction to supersymmetric $\sigma$-models is also rather straightforward and it will be presented elsewhere.

\section{Acknowledgements}

We would like to thank C. Burgess, E. Kiritsis, F.Quevedo, G. Veneziano and E. Verlinde for valuable discussions. E.A. and Y.L. were supported in part by the CICYT grant AEN 93/673 (Spain) and by a fellowship of Comunidad Autónoma de Madrid (YL).

\section{Note added}

After this work was completed we learned of the work [28] where another attempt to understand non-abelian duality is made. We also learned that in the note added to [5] they independently pointed out that the possible violation of conformal invariance in the model of [8] should be related to the fact that for non-semisimple groups there may be generators in the adjoint representation whose trace is not zero. We would like to thank M. Rocek for bringing this information to our attention. 


\section{References}

[1] J.M. Drouffe and C. Itzykson, Quantum field theory and statistical mechanics (Cambridge University Press, 1990).

[2] K. Kikkawa and M. Yamasaki, Phys. Lett. B149 (1984) 357; N. Sakai and I. Senda, Prog. Theor. Phys. 75 (1984) 692; T.H. Buscher, Phys. Lett. B194 (1987)51, B201(1988) 466; V.P. Nair, A. Shapere, A. Strominger and F. Wilczek, Nucl. Phys. B287 (1987) 402; A. Shapere and F. Wilczek, Nucl. Phys. B320 (1989) 609; A. Giveon, E. Rabinovici and G. Veneziano, Nucl. Phys. B322 (1989) 167; E. Álvarez and M.A.R. Osorio, Phys. Rev. D40 (1989) 1150; E. Kiritsis, Nucl. Phys. B405 (1993) 109; A. Giveon and E. Kiritsis, Nucl. Phys. B411 (1994) 487; A. Giveon, M. Porrati and E. Rabinovici, Target space duality in string theory, NYU-TH-94/01/01.

[3] X. de la Ossa and F. Quevedo, Nucl. Phys. B403 (1993) 377.

[4] E. Álvarez, L. Álvarez-Gaumé, J.L.F. Barbón and Y. Lozano, Nucl. Phys. B415 (1994) 71; L. Álvarez-Gaumé, Aspects of abelian and nonabelian duality, CERN-TH-7036/93.

[5] A. Giveon and M. Roček, On Nonabelian Duality, ITP-SB-93-44.

[6] M. Roček and E. Verlinde, Nucl. Phys. B373 (1992) 630.

[7] P. Goddard, A. Kent and D. Olive, Comm. Math. Phys. 103 (1986) 105.

[8] M. Gasperini, R. Ricci and G. Veneziano, Phys. Lett. B319 (1993) 438.

[9] L. Álvarez-Gaumé and E. Witten, Nucl. Phys. B234 (1984) 269.

[10] E. Witten, Comm. Math. Phys. 92 (1984) 455.

[11] See, for example: T. Eguchi, P. Gilkey and A. Hanson, Phys. Rep. 66 (1980) 213, and references therein.

[12] C. Hull and B. Spence, Phys. Lett B232 (1989) 204.

[13] I. Jack, D.R.T. Jones, A. Mohammedi and H. Osborn, Nucl. Phys. B332 (1992) 359. 
[14] C. Callan, D. Friedan, E. Martinec and M. Perry, Nucl. Phys. B262 (1985) 593; C. Callan, I. Klebanov and M. Perry, Nucl. Phys. B278 (1986) 78.

[15] D. Friedan, E. Martinec and S. Shenker, Nucl. Phys. B271 (1986) 93.

[16] P.B. Gilkey, J. Diff. Geom. 10 (1975) 601.

[17] M. Douglas, Ph.D. Thesis, CalTech 68-1453.

[18] A. M. Polyakov and P.B. Wiegmann, Phys. Lett. B131 (1983) 121, B141(1984) 223.

[19] K. Bardacki, E. Rabinovici and B. Säring, Nucl. Phys. B299 (1988) 157; D. Altschuler, K. Bardacki and E. Rabinovici, Comm. Math Phys. 118 (1988) 241; K. Gawedzki and A. Kupiainen, Phys. Lett. B215 (1988) 119, Nucl. Phys. B320 (1989) 625; D. Karabali, Q.H. Park, H. Schnitzer and Z. Yang, Phys. Lett B216 (1989) 307; D. Karabali and H. Schnitzer, Nucl. Phys. B329 (1990) 649.

[20] E. Witten, Phys. Rev D44 (1991) 314.

[21] R. Dijkgraaf, E. Verlinde and H. Verlinde, Nucl. Phys. B371 (1992) 269.

[22] A. Tseytlin, Nucl. Phys. B399 (1993) 601.

[23] A. Giveon and M. Roček, Nucl. Phys. B380 (1992) 128.

[24] Work in progress.

[25] R. Dijkgraaf and E. Witten, Comm. Math. Phys. 129 (1990) 393.

[26] M. B. Green, J. Schwarz and E. Witten, Superstring Theory (Cambridge University Press, 1987).

[27] T. Eguchi and A. Hanson, Ann. Phys. 120 (1979) 82; G. Gibbons and S. Hawking, Comm. Math. Phys. 66 (1979) 291.

[28] K. Sfetsos, Gauged WZW models and non-abelian duality, THU-94/01. 